\title{
Relationship between Abdominal Strength Measured by a Newly Developed Device and Abdominal Muscle Thickness
}

\author{
Takanori Noguchi' ${ }^{1}$, Shinichi Demura² \\ ${ }^{1}$ Fukui University of Technology, Fukui, Japan \\ ${ }^{2}$ Graduate School of Natural Science \& Technology, Kanazawa University, Kanazawa, Japan \\ Email: t-noguchi@fukui-ut.ac.jp
}

Received 16 December 2013; revised 16 January 2014; accepted 23 January 2014

Copyright (C) 2014 by authors and Scientific Research Publishing Inc.

This work is licensed under the Creative Commons Attribution International License (CC BY).

http://creativecommons.org/licenses/by/4.0/

(c) (i) Open Access

\begin{abstract}
Although measurement of abdominal strength is important for evaluating trunk myofunction, adequate devices for these measurements have not been developed. As a consequence, the relationships between abdominal strength and abdominal muscle thickness have also not been determined. This study aimed to develop a new device for measuring abdominal strength and to examine the relationship between abdominal muscle strength and thickness. The subjects in the study included 50 young males (age, $19.1 \pm 0.32$ years; height, $171.0 \pm 6.55 \mathrm{~cm}$; weight, $69.5 \pm$ $12.15 \mathrm{~kg}$ ). The measurement of abdominal flexion strength had high reliability with an intra-class correlation coefficient (ICC) $>0.9$. The thickness of the rectus abdominis muscle was measured under two conditions using an ultrasound imaging method; relaxation and strength exertion. Abdominal muscle thickness was significantly thicker during exertion than during relaxation. Abdominal flexion strength showed a significant correlation with muscle thickness during both relaxation ( $r=0.69$ ) and strength exertion $(r=0.71)$. No significant difference was found between these correlation coefficients. In conclusion, the values of abdominal strength determined by our newlydeveloped measuring device had high reliability, with abdominal flexion strength showing a relatively close relationship with the thickness of the rectus abdominis during strength exertion and relaxation.
\end{abstract}

Keywords

Ultrasound Imaging Method, Measurement Device, Strength Exertion

\section{Introduction}

Abdominal muscle groups contribute to enhanced intra-abdominal pressure, thereby stabilizing the vertebral

How to cite this paper: Noguchi, T., \& Demura, S. (2014). Relationship between Abdominal Strength Measured by a Newly Developed Device and Abdominal Muscle Thickness. Advances in Physical Education, 4, 70-76.

http://dx.doi.org/10.4236/ape.2014.42010 
column, and maintaining posture (Michael, Erik, \& Udo, 2010). Therefore, their development is important, not only for athletes, but also for the general population. It is absolutely necessary to maintain and increase abdominal strength in order for the rectus abdominis muscle to function properly during the activities of daily living. However, it is difficult to measure abdominal flexion strength of the rectus abdominis muscle, and useful devices to easily measure parameters such as grip or leg strength have not been developed. It is therefore necessary to develop reliable devices for measuring abdominal strength.

Muscle thickness is related closely with muscle mass (Akagi, Takai, Kato, Fukuda, Wakahara, Ohta, Kanehisa, Kawakami, \& Fukunaga, 2009; Noguchi, Demura, \& Takahashi, 2013) and is also considered to be associated with strength exertion of related body parts. Miyamoto et al. (2007) reported that the thickness of the rectus femoris muscle was related closely to knee extension strength. However, the rectus abdominis muscle has a more different form than the rectus femoris muscle. As a consequence of the lack of useful measurement devices, the relationship between the rectus abdominis muscle and muscle thickness has not been examined.

Ultrasound imaging is used routinely to measure muscle thickness (Koppenhaver, Hebert, Fritz, Parent, Teyhen, \& Magel, 2009; Ogawa, Mitsukawa, Bemben, \& Abe, 2012; Noguchi, Demura, \& Takahashi, 2013; Teyhen, 2006; Teyhen, 2007), with the reliability of measurements for skinfold or muscle thickness being very high (Mannion, Pulkovski, Toma, \& Sprott, 2008; Weits, Van der Beek, \& Wedel, 1986). It is also possible to easily measure the thickness of the rectus abdominis muscle using a smaller and lighter device that was recently developed.

Because fat is an inactive tissue, measurements do not change (Michael, Erik, \& Udo, 2010). In contrast, muscle thickness changes according to the level of strength exertion, such as during maximal exertion and complete relaxation.

The following questions need to be answered:

a) To what degree does rectus abdominis thickness differ during the exertion and relaxed states?

b) What relationships are found between abdominal flexion strength and abdominis thickness in the above two states, and which state is more closely related to muscle strength?

Clarifying these two questions may provide useful information to explain the relationships between anatomical (muscle thickness) and functional characteristics (muscle strength) of the rectus abdominis.

The purpose of this study was to develop a useful device for measuring abdominal flexion strength and to investigate the relationship between muscle strength and thickness during relaxation and exertion.

\section{Methods}

\subsection{Subjects}

The subjects in the study included 50 young, healthy males (age, $19.1 \pm 0.32$ years; height, $171.0 \pm 6.55 \mathrm{~cm}$; weight, $69.5 \pm 12.15 \mathrm{~kg}$ ). The purpose and procedures of this study were explained in detail to all participants before the experiment. Written, informed consent was obtained prior to enrollment in the study. The experimental protocol was approved by the Ethics Committee on Human Experimentation of the Faculty of Human Science, Kanazawa University (No. 2012-14).

\subsection{Procedures}

Abdominal flexion strength and muscle thickness were measured in the study.

1) Abdominal flexion strength test

A device for measuring trunk strength was used to measure abdominal flexion strength (original model: Takei Scientific Instruments Co. Ltd. Tokyo, Japan). The device consists of the main body of an abdominal crunch machine and a strain gauge (T.K.K.1268, Takei Scientific Instruments Co., Ltd., Tokyo, Japan) (Figure 1). The measuring range of the strain gauge is $0-100 \mathrm{~kg}$, and the measurement error is $\pm 0.5 \% \mathrm{FS}$ or less.

The strength exerted was measured by a strain-gauge meter connected through a pulley, whilst the subject pushed a rotation lever at chest height. Zero adjustment was conducted such that the rotary lever was untouched. After adjusting the height of the chair to match the seated height of the subject, the subjects were requested to sit so that their abdominal flexion point matched the rotation axis of the lever. The subjects placed both elbows on the base of the rotation lever with their arms folded and then exerted abdominal flexion strength maximally. At the time of maximal exertion, a researcher checked whether the subject had not pushed the lever with their hands 


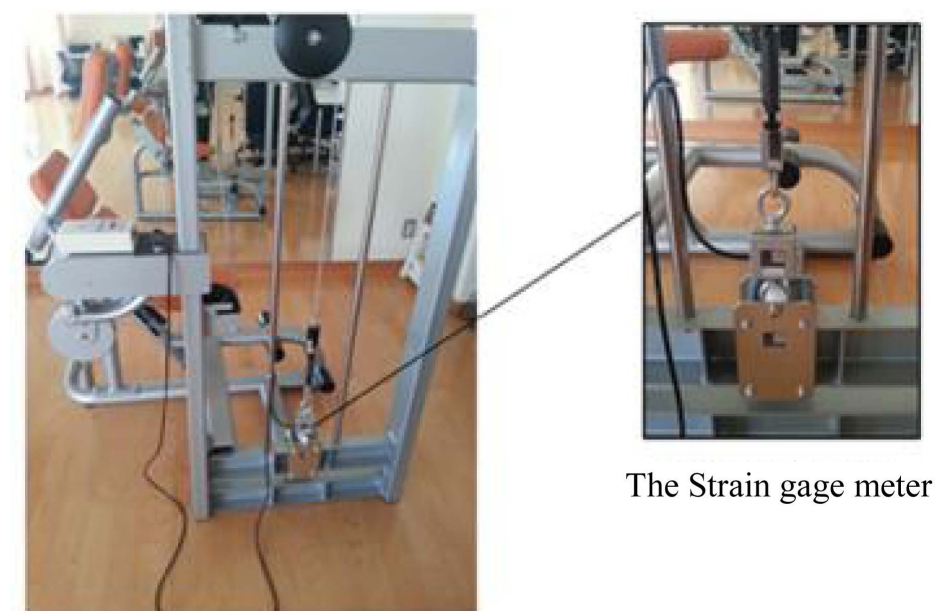

Figure 1. The device for measuring trunk strength.

or arms or had leaned back. The values measured by the strain-gauge in the pulley were revised using the following formula:

$$
\text { Abdominal flexion strength }=\text { measurement value }(\mathrm{kg}) \times 0.6
$$

After one practice, two measurements of strength were recorded. A 1 min rest period was taken between each trial to eliminate the effect of fatigue.

2) Thickness of Abdominal Muscles

An ultrasound imaging device (GT-101, TANITA, Tokyo, Japan) was used to measure the thickness of the abdominal muscles. Figure 2 shows computer displayed ultrasound images using the B-mode method. The thickness of the rectus abdominis muscle was measured using a probe frequency of $6 \mathrm{MHz}$ at the maximal width of a point 4-cm transverse from the navel, excluding the tendinous intersections (Figure 3). All measurements were performed by an experienced researcher. The thickness of each abdominal muscle was measured twice in the upright standing position under the following two conditions.

- Relaxation state

A state that relieves abdominal pressure without the rectus abdominis muscle exerting strength.

- Strength exertion state

A state that exerts abdominal strength to increase abdominal pressure by holding one's breath that causes contraction of the rectus abdominis. Palpation was used to confirm that the subjects had performed the relaxed and strength exertion conditions properly. Measurements of muscle thickness were then recorded during two alternate series of relaxation and exertion. The difference between relaxed and strength exertion muscle thickness was examined.

\subsection{Statistical Analyses}

The intra-class correlation coefficient (ICC) was calculated to evaluate trial-to-trial reliability. A paired t test was used to compare the difference between mean muscle thicknesses during relaxation and strength exertion. The relationship between abdominal flexion strength and both abdominal muscle thicknesses was examined using Pearson's correlation coefficient. Statistical significance $(\alpha)$ was set at $p<0.05$.

\section{Results}

Table 1 shows the ICCs for measurement of abdominal flexion strength and muscle thickness during relaxation and strength exertion, and mean differences between mean muscle thicknesses during relaxation and exertion.

All variables showed very high ICCs $^{s}$ of $>0.98$. Figure 4 and Figure 5 show the correlation between each abdominal muscle thickness and abdominal flexion strength. Abdominal flexion strength showed a significant correlation with muscle thickness during both relaxation $(r=0.70)$ and strength exertion $(r=0.70)$. The correlation coefficients were not significantly different. 


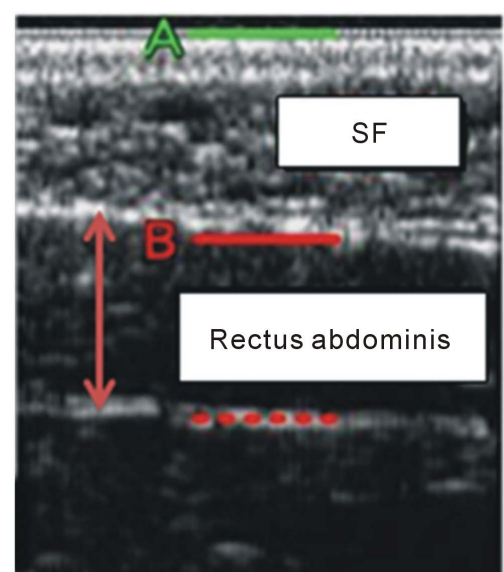

Figure 2. Measurement of muscle thickness.

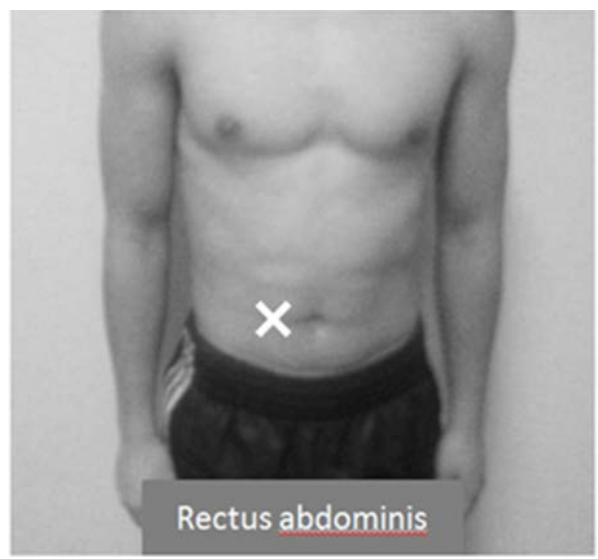

Figure 3. Measurement region.

Table 1. ICCs of abdominal flexion strength, muscle thickness during relaxation and strength exertion, and their means, and a test result between means.

\begin{tabular}{ccccccccccccc}
\hline & \multicolumn{1}{c}{ 1st. } & \multicolumn{9}{c}{ 2nd. } & \multicolumn{7}{c}{ Average } \\
\hline $\mathrm{N}=50$ & unit & Mean & SD & Mean & SD & ICC & F value & Mean & SD & t & $p$ value \\
\hline Relaxation & $(\mathrm{mm})$ & 15.7 & 2.45 & 15.8 & 2.49 & 0.98 & 0.01 & 15.7 & 2.46 \\
Exertion & $(\mathrm{mm})$ & 17.8 & 3.28 & 17.8 & 3.35 & 0.99 & 0.00 & 17.8 & 3.31 & 9.18 & $*$ & 0.00 \\
Difference & $(\mathrm{mm})$ & - & - & - & - & - & - & 2.0 & 1.56 & & \\
Flexion strength & $(\mathrm{kg})$ & 46.4 & 11.85 & 46.5 & 11.82 & 0.99 & 0.01 & & & & \\
\hline
\end{tabular}

Note: ${ }^{*} p<0.05$.

\section{Discussion}

This first aim of this study was to develop a useful device for measuring abdominal flexion strength, and then to examine the relationship between abdominal strength and abdominal muscle thickness.

\subsection{Reliability of Abdominal Flexion Strength Measurement Device}

Good reliability is important to ensure accuracy and consistency of devices for measuring muscle strength. In particular, when developing a new device, it is essential that its reliability is examined. Until now, devices to directly measure abdominal flexion strength have not been developed.

However, exercise machines designed for abdominal flexion exercises have recently become popular in training facilities as they make it possible to exert abdominal flexion strength in a prescribed manner. The device 


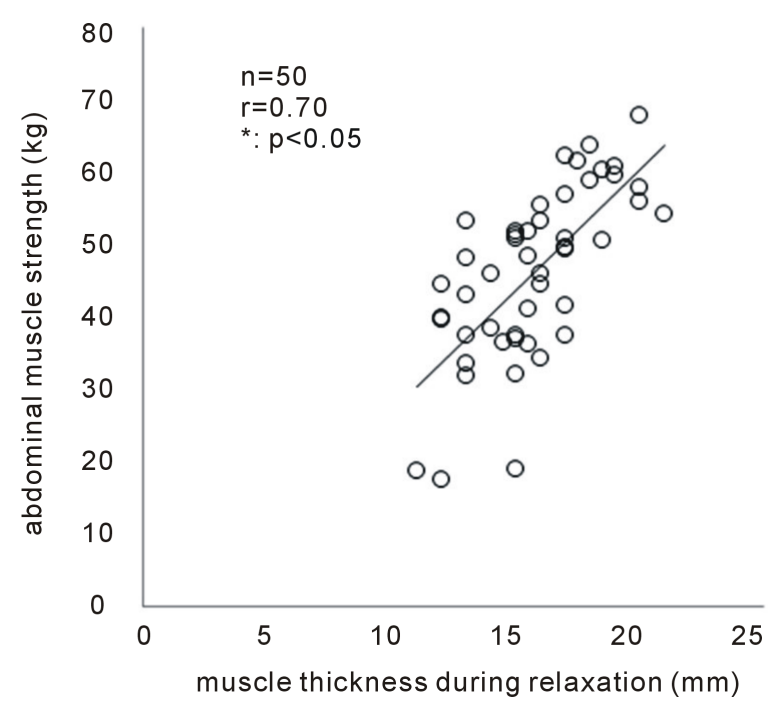

Figure 4. The correlation between abdominal muscle thicknesses during relaxation and abdominal flexion strength.

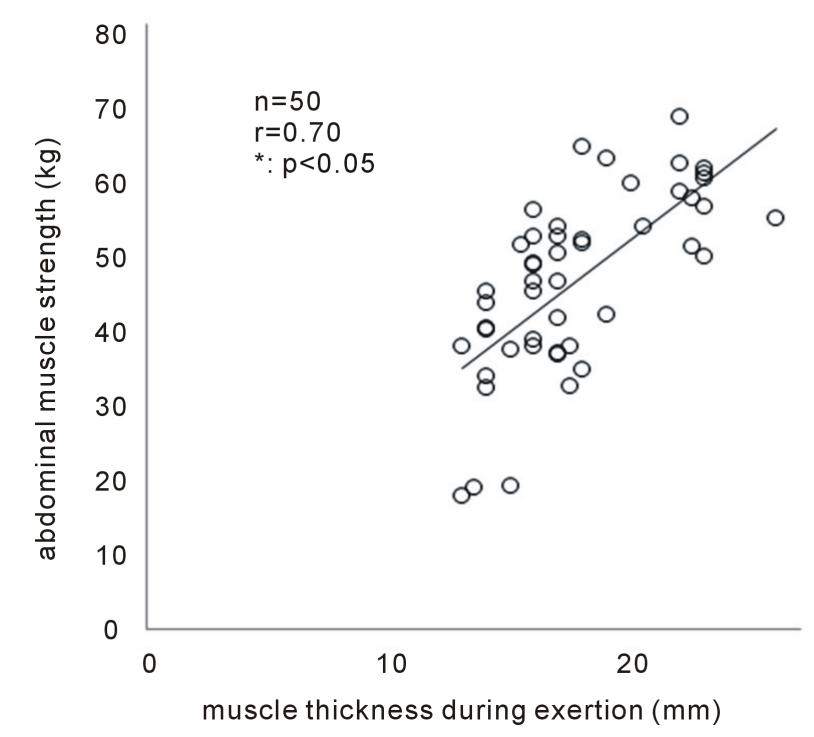

Figure 5. The correlation between abdominal muscle thicknesses during exertion and abdominal flexion strength.

developed in this study was a remodeled version of this machine. The reliability of the measurements (ICC range 0.98 - 0.99) was considerably higher than that for knee extension and flexion strength values (ICC range 0.76 - 0.97) developed by Koblbauer et al. (2011). We therefore considered that the present device is useful for measuring abdominal flexion strength.

However, this device has the following weak points and limitations: the size of the measurement device is too large and it is not easily portable; precise measurement is not possible when the subject's posture is improper; and consistency has to be checked when measurements are made using other machines.

It is possible that this device may enter general use for measuring abdominal muscle power in the future if these weak points and limitations are improved.

\subsection{Change in Muscle Thickness}

Unlike subcutaneous fat tissues, muscle tissues change thickness depending on the state of strength exertion. 
Miyamoto et al. (2007) reported that the thickness of the rectus femoris muscle increased by a mean $3.9 \mathrm{~mm}$ during strength exertion (mean, $20.2 \mathrm{~mm}$ ) compared with relaxation (mean, $16.3 \mathrm{~mm}$ ). Similarly, our study showed a mean increase of $2.6 \mathrm{~mm}$ in the rectus abdominis thickness during strength exertion, with a significant difference between both muscle states. Although the rectus abdominis selected in this study shows smaller differences between exertion and relaxation compared with the rectus femoris, we clarified that it becomes thicker during strength exertion, similar to that observed in other muscles. Accordingly, when muscle thickness differs according to the level of exertion, it is necessary to clarify whether muscle thickness is related more closely to strength.

\subsection{Abdominal Muscle Thickness and Abdominal Flexion Strength}

We observed a significant correlation between abdominal muscle thicknesses during strength exertion and abdominal flexion strength. Miyamoto et al. (2007) reported that knee extension strength is related to the combined muscle thickness of the rectus femoris and vastus intermedius muscles during strength exertion $(r=0.62)$. The correlations measured in the current study were somewhat higher $(r=0.69,0.71)$. In contrast to Miyamoto et al., we also showed a significant correlation between muscle thickness and strength even in the relaxed state. As stated above, because the change in muscle thickness between exertion and relaxation is smaller in the rectus abdominis muscle than in the femoral area, it is inferred that muscle thickness even in the relaxed state shows a significant relationship with abdominal flexion strength. However, the strength of this relationship was not as high as that measured during exertion $\left(\mathrm{R}^{2}=42 \%-53 \%\right)$. The two following points are considered as possible explanations for this difference.

1) Maximal strength is not determined only by the cross-sectional area of muscle, but also by other factors such as mobilization ratio and type (ratio) of muscle fibers.

2) The measurements of abdominal rectus muscle may not be sufficient to estimate abdominal flexion strength. In short, other muscles such as the abdominal internal and external oblique muscles may also have participated in abdominal flexion.

It may also be necessary to measure multiple parts because the abdominal rectus muscle itself is polygastric. These limitations should therefore be taken into account when examining the relationships between abdominal muscle thickness and strength. Notwithstanding these limitations, our study provided useful information for evaluating the effectiveness of training as it showed that there is a significant relationship between abdominal strength, which is difficult to measure directly, and muscle thickness.

\section{Acknowledgements}

This work was supported by Japan Society for the Promotion of Science (JSPS) KAKENHI Grant-in-Aid for Young Scientists (B) Number 24700673.

\section{References}

Akagi, R., Takai, Y., Kato, E., Fukuda, M., Wakahara, T., Ohta, M., Kanehisa, H., Kawakami, Y., \& Fukunaga, T. (2009). Relationships between Muscle Strength and Indices of Muscle Cross-Sectional Area Determined during Maximal Voluntary Contraction in Middle-Aged and Elderly Individuals. Journal of Strength and Conditioning Research, 23, $1258-1262$. http://dx.doi.org/10.1519/JSC.0b013e3181918a9b

Koblbauer, I. F., Lambrecht, Y., Van der Hulst, M. L., Neeter, C., Engelbert, R. H., Poolman, R. W., \& Scholtes, V. A. (2011). Reliability of Maximal Isometric Knee Strength Testing with Modified Hand-Held Dynamometry in Patients Awaiting Total Knee Arthroplasty: Useful in Research and Individual Patient Settings? A Reliability Study. BMC Musculoskeletal Disorders, 12, 249. http://dx.doi.org/10.1186/1471-2474-12-249

Koppenhaver, S. L., Hebert, J. J., Fritz, J. M., Parent, E. C., Teyhen, D. S., \& Magel, J. S. (2009). Reliability of Rehabilitative Ultrasound Imaging of the Transversus Abdominis and Lumbar Multifidus Muscles. Archives of Physical Medicine and Rehabilitation, 90, 87-94. http://dx.doi.org/10.1016/j.apmr.2008.06.022

Mannion, A. F., Pulkovski, N., Toma, V., \& Sprott, H. (2008). Abdominal Muscle Size and Symmetry at Rest and during Abdominal Hollowing Exercises in Healthy Control Subjects. Journal of Anatomy, 213, 173-182. http://dx.doi.org/10.1111/j.1469-7580.2008.00946.x

Michael, S., Erik, S., \& Udo, S. (2010). Prometheus: Lernatlas der Anatomie (pp. 130-141). Tokyo: Igakusyoin.

Miyamoto, K., Tanaka, S., Tanaka, A., Matsumura, S., Tanisawa, T., Moriyama, S., Nakatani, T., \& Tanaka, S. (2007). Ap- 
propriation of Colorized Ultrasonographics, and Relationships between Muscle Thickness and Muscle Volume or Strength. Structure and Function, 6, 27-32.

Ogawa, M., Mitsukawa, N., Bemben, M. G., \& Abe, T. (2012). Ultrasound Assessment of Adductor muscle Size Using Muscle Thickness of the Thigh. Journal of Sport Rehabilitation, 21, 244-248.

Noguchi, T., Demura, S., \& Takahashi, K. (2013). Relationships between Sit-Ups and Abdominal Flexion Strength Tests and the Thickness of Each Abdominal Muscle. Advanced in Physical Education, 3, 84-88. http://dx.doi.org/10.4236/ape.2013.32014

Teyhen, D. S. (2006). Rehabilitative Ultrasound Imaging Symposium. Journal of Orthopaedic and Sports Physical Therapy, 36, A-1-A-17. http://dx.doi.org/10.2519/jospt.2006.0301

Teyhen, D.S. (2007). Rehabilitative Ultrasound Imaging: The Roadmap Ahead. Journal of Orthopaedic and Sports Physical Therapy, 37, 431-433. http://dx.doi.org/10.2519/jospt.2007.0107

Weits, T., Van der Beek, E. J., \& Wedel, M. (1986). Comparison of Ultrasound and Skinfold Caliper Measurement of Subcutaneous Fat Tissue. International Journal of Obesity, 10, 161-168. 